\title{
COHOMOLOGY AS THE DERIVED FUNCTOR OF DERIVATIONS
}

\author{
BY \\ MICHAEL BARR(1) AND GEORGE S. RINEHART(2)
}

Introduction. The investigations which produced this paper were suggested by the fact that the Hochschild cohomology $\hat{H}(F, M)$ of a free algebra $F$, with coefficients in any module $M$, is zero in dimension $\geqq 2$. Since free algebras are projectives in the category of algebras, this suggested that $\hat{H}(-, M)$, considered as a functor of the first variable, ought to resemble a derived functor. We have in fact obtained one further resemblance: the existence, corresponding to any exact sequence of algebras split over the ground ring, of a "connecting homomorphism", in the first variable, and the exactness of the resulting infinite sequence. The prerequisite for this is the remark that, given an algebra $R, \hat{H}(R, M)$ is not only obtainable as a derived functor in the usual way $[1, \mathrm{IX}]$, but also, suitably renumbered, as the derived functor in the category of $R$-modules of $\operatorname{Der}(R, M)$, the derivations from $R$ to $M$.

The existence of the connecting homomorphism allows one to obtain a functorial proof of the correspondence between second cohomology and extensions which is entirely analogous to the proof for modules found in $[1, \mathrm{XIV}, \S 1]$, and to extend this correspondence to one of the Yoneda type for higher cohomology.

Similar arguments and results obtain for supplemented algebras, Lie algebras, and groups, and we have presented these simultaneously.

We conclude the paper with a proof that, in degree $\geqq 2$, the cohomology of the free product of two groups is the direct sum of the cohomologies of each of them.

Some of our terminology is based implicitly on Eilenberg and Moore's notion of projective and injective classes [2].

1. Cohomology as the derived functor of derivations. Let $K$ be a commutative ring with unit. In what follows, $\mathscr{C}$ will denote one of the following categories:

Received by the editors January 8, 1964 and, in revised form, March 22, 1965.

(1) This work has been partially supported by the National Science Foundation under Grant NSF GP-1904.

(2) Research for this paper was carried out while this author held a postdoctoral fellowship from the National Academy of Sciences-National Research Council, funds for which were supplied by the Air Force Office of Scientific Research. 
Case A. Unitary $K$-algebras.

Case S. Supplemented unitary $K$-algebras; that is, unitary $K$-algebras with a unitary $K$-algebra supplementation homomorphism to $K$. Morphisms in the category are unitary $K$-algebra maps which commute with the supplementation.

Case L. Lie algebras with $K$ as ground ring.

Case G. Groups (not necessarily commutative).

If $R \in \mathscr{C}, \mathscr{M}_{R}$ will denote the following category.

Case A. Two sided $R$-modules.

Case S. Left $R$-modules. $M$ is given the structure of a right $R$-module defined by $m r=\varepsilon(r) m$ where $\varepsilon: R \rightarrow K$ is the supplementation.

Case L. Left modules for the $K$-Lie algebra $R$. If $M \in \mathscr{M}_{R}$, we will give $M$ the structure of a right $R$-module defined by $m r=-r m$, for $m \in M, r \in R$.

Case G. Left $R$-modules. If $M \in \mathscr{M}_{R}$, we will give $M$ the trivial structure as a right $R$-module; i.e., $m r=m$, for $m \in M, r \in R$.

The abelian group of morphisms between $M$ and $M^{\prime}$ in $\mathscr{M}_{R}$ will be denoted by $\operatorname{Hom}_{R}\left(M, M^{\prime}\right)$.

Let $\mathscr{Q}$ denote the class of all monomorphisms in $\mathscr{M}_{R}$ in Case $\mathrm{G}$, and all $K$-split monomorphisms (monomorphisms which have a $K$-linear left inverse) in $\mathscr{M}_{R}$ in Cases A, S and L. We say that $Q \in \mathscr{M}_{R}$ is $\mathscr{Q}$-injective in case, whenever $f: M \rightarrow M^{\prime}$ is in $\mathscr{Q}$, the map from $\operatorname{Hom}_{R}\left(M^{\prime}, Q\right)$ to $\operatorname{Hom}_{R}(M, Q)$ induced by $f$ is surjective. We say that a short sequence $0 \rightarrow M^{\prime} \rightarrow^{f} M \rightarrow M^{\prime \prime} \rightarrow 0$ is 2 -exact in case it is exact and $f \in \mathscr{Q}$. Other sequences are $\mathscr{Q}$-exact if they are made up from short $\mathcal{Q}$-exact sequences in the obvious way. In each of our cases, given $R \in \mathscr{C}$, there is a ring $R^{e}$ with unit such that every $R$-module has a natural structure as a unitary left $R^{e}$-module and conversely. Hence it follows (see [1] and [3]) that there are enough $\mathscr{Q}$-injectives, in the sense that for every $M \in \mathscr{M}_{R}$ there is an element $f: M \rightarrow Q$ in $\mathscr{Q}$, where $Q$ is a $\mathscr{Q}$-injective. One uses this to obtain $\mathscr{Q}$-exact $\mathscr{Q}$-injective resolutions and derived functors in the usual fashion.

Let $\phi: T \rightarrow R$ be a morphism in $\mathscr{C}$, and let $M \in \mathscr{M}_{R}$. Define $\operatorname{Der}_{\phi}(T, M)$ $=\left\{f \in \operatorname{Hom}_{K}(T, M) \mid f(x y)=\phi(x) f(y)+f(x) \phi(y)\right\}$, where in case $G, \operatorname{Hom}_{K}$ denotes set mappings. Consider $\operatorname{Der}_{\phi}(T,-)$ as a functor from $\mathscr{M}_{R}$ to abelian groups and let $H_{\phi}(T,-)$ be its $n$th derived functor using the class $\mathscr{Q}$ as described above. In case $\phi: R \rightarrow R$ is the identity, we will write $\operatorname{Der}(R, M)$ for $\operatorname{Der}_{\phi}(R, M)$ and $H(R, M)$ for $H_{\phi}(R, M)$.

Proposition 1.1. $H_{\phi}^{0}(T, M)=\operatorname{Der}_{\phi}(T, M)$.

Proof. This follows at once from the easily established fact that $\operatorname{Der}_{\phi}(T,-)$ is left exact.

Definition 1.1. For $M \in \mathscr{M}_{R}$, let $M \times R$ be the direct product of $M$ and $R$ as $K$-modules (sets in Case G). Then $M \times R$ may be considered as an object in $\mathscr{C}$ by defining $(m, r)\left(m^{\prime}, r^{\prime}\right)=\left(m r^{\prime}+r m^{\prime}, r r^{\prime}\right)$. Moreover, there is a natural morphism 
$\phi_{0}: M \times R \rightarrow R$ and a natural 1-1 correspondence between $\operatorname{Der}_{\phi}(T, M)$ and $\left\{\alpha: T \rightarrow M \times R \mid \phi_{0} \alpha=\phi\right\}$.

Let $\mathscr{C}_{0}$ be the category of sets in Case G, of unitary $K$-modules in Cases A and $\mathrm{L}$, and of "supplemented" unitary $K$-modules (in the obvious sense) in Case S. Then there is a natural inclusion functor of $\mathscr{C}$ into $\mathscr{C}_{0}$. Moreover, this functor has a left adjoint; i.e. there is a functor $F: \mathscr{C}_{0} \rightarrow \mathscr{C}$ and an isomorphism of bifunctors $\mathscr{C}_{0}(-,-) \cong \mathscr{C}(F(-),-)$, where $\mathscr{C}(T, R)$ denotes morphisms from $T$ to $R$ in $\mathscr{C}$. In Cases $A$ and $S, F(U)$ is the tensor algebra on $U$; in Case $L$ the free Lie algebra on $U$, defined as the appropriate homomorphic image of the nonassociative tensor algebra on $U[1$, p. 285, Example 4]; in Case $G$ the free group on $U$. Note that the adjointness relationship implies that $F(U)$ is a projective for the class of all $K$-split surjections (or of all surjections in Case $\mathrm{G}$ ).

Suppose $\phi \in \mathscr{C}(F(U), R)$ and $\phi^{\prime} \in \mathscr{C}_{0}(U, R)$ are corresponding morphisms. By the above we have $\operatorname{Der}_{\phi}(F(U), M)=\left\{\alpha \in \mathscr{C}(F(U), L M \times R) \mid \phi_{0} \alpha=\phi\right\}$ $=\left\{\alpha^{\prime} \in \mathscr{C}_{0}(U, M \times R) \mid \phi_{0} \alpha^{\prime}=\phi^{\prime}\right\}$. But $M \times R$ is just the product of $M$ and $R$ in $\mathscr{C}_{0}$, and $\phi_{0}$ is the projection morphism to $R$. Hence $\operatorname{Der}_{\phi}(F(U), M)$ may be identified with $\mathscr{C}_{0}(U, M)$. But $\mathscr{C}_{0}(U,-)$ is exact on 2 -exact sequences. Hence we have

Proposition 1.2. $H_{\phi}^{n}(F(U), M)=0$ for $n \geqq 1$.

For $N \in \mathscr{M}_{R}$, let $\operatorname{Ext}_{R}(N,-)$ be the derived functor, using $\mathscr{Q}$, of the functor $\operatorname{Hom}_{R}(N,-)$. Then, in the notation of [3], $\operatorname{Ext}_{R}(N, M)=\operatorname{Ext}_{\left(R^{e}, K\right)}(N, M)$, except in Case $\mathrm{G}$, where $\operatorname{Ext}_{R}(N, M)=\operatorname{Ext}_{R^{e}}(N, M)$.

Let $B \in \mathscr{M}_{R}$ be $R$ in Case A, $K$ with the module structure defined via the augmentation in Case S, $K$ with trivial operation $(R \cdot K=\{0\})$ in Case L, and the integers with trivial operation (elements of $R$ act as the identity homomorphism) in Case G. Let $\hat{H}(R, M)=\operatorname{Ext}_{R}(B, M)$. Then $\hat{H}(R, M)$ is the usual cohomology of the algebra (respectively Lie algebra, group) $R$ with coefficients in $M$. (See [1] and [3].)

There is a 2 -exact sequence $0 \rightarrow I \rightarrow R^{e} \rightarrow B \rightarrow 0$, and the functors $\operatorname{Hom}_{R}(I,-)$ and $\operatorname{Der}(R,-)$ are naturally equivalent $[1, \mathrm{pp} .168,183,270]$. Hence $\operatorname{Ext}_{R}(I, M)$ $=H(R, M)$; and, for $n \geqq 1$, we have exact sequences

$$
0=\operatorname{Ext}_{R}^{n}\left(R^{e}, M\right) \rightarrow \operatorname{Ext}_{R}^{n}(I, M) \rightarrow \operatorname{Ext}_{R}^{n+1}(B, M) \rightarrow \operatorname{Ext}_{R}^{n+1}\left(R^{e}, M\right)=0 .
$$

Hence

Proposition 1.3. $H^{n}(R, M) \cong \hat{H}^{n+1}(R, M)$ if $n \geqq 1$.

2. The connecting homomorphism in the first variable. Let $\phi: T \rightarrow R$ be a surjection in $\mathscr{C}$ with kernel $S$. In Cases $A$ and $\mathrm{L}$, define $S^{2}$ to be the ideal generated by $\{x y \mid x, y \in S\}$. In Case $S$, let $I$ be the kernel of the supplementation map from $T$ to $K$, and let $S^{2}=S I$. In Case G, let $S^{2}$ be the commutator subgroup of $S$. $S / S^{2}$ has the natural structure of an abelian group. $T$ acts on $S$ by multiplication 
(except in Case $\mathrm{G}$ where the action is $s \rightarrow t s t^{-1}, s \in S, t \in T$ ), and this action sends $S^{2}$ into itself. Thus $S / S^{2}$ has a $T$-module structure, and the action of $S$ on this module is trivial. Thus we may regard $S / S^{2}$ as an object in $\mathscr{M}_{R}$.

If $M \in \mathscr{M}_{R}$, there is a natural exact sequence of abelian groups

$$
0 \rightarrow \operatorname{Der}(R, M) \rightarrow \operatorname{Der}_{\phi}(T, M) \rightarrow \operatorname{Hom}_{R}\left(S / S^{2}, M\right)
$$

in which the last map is induced by restriction from $T$ to $S$.

THEOREM 2.2. Let $\phi: T \rightarrow R$ be a surjection in $\mathscr{C}$ with kernel $S$, and let $M \in \mathscr{M}_{R}$. In Cases A and $\mathrm{S}$, assume that $\phi$ is $K$-split (has a K-linear right inverse); and in Case L assume that $R$ is $K$-projective. Define $\operatorname{Ext}_{R}$ as at the end of $\S 1$. Then, for all $n \geqq 0$, there is a connecting homomorphism from $\operatorname{Ext}_{R}^{n}\left(S / S^{2}, M\right)$ to $H^{n+1}(R, M)$, such that the sequence

$$
\cdots \rightarrow H^{n}(R, M) \rightarrow H_{\phi}^{n}(T, M) \rightarrow \operatorname{Ext}_{R}^{n}\left(S / S^{2}, M\right) \rightarrow H^{+1}(R, M) \rightarrow \cdots
$$

is exact, where the remaining maps are induced by those of (2.1).

Proof. Note first that since every derivation from $T$ to an $R$-module vanishes on $S^{2}$, we may replace $S$ and $T$ by $S / S^{2}$ and $T / S^{2}$ respectively, and hence may assume without loss of generality that $S^{2}=0$. (In Case G ' 0 ', will be used to denote the identity.)

We will define an object $D_{\phi}(T)$ of $\mathscr{M}_{R}$ with the property that the functors $\operatorname{Hom}_{R}\left(D_{\phi}(T),-\right)$ and $\operatorname{Der}_{\phi}(T,-)$ are isomorphic. We will also define a natural morphism $j: S \rightarrow D_{\phi}(T)$ in $\mathscr{M}_{R}$. If we write $D(R)=D_{\phi}(R)$ for $\phi$ the identity map on $R$, there will be an obvious epimorphism $D_{\phi}(T) \rightarrow D(R)$. Moreover, it can be readily verified that this epimorphism induces an isomorphism between $D_{\phi}(T) / j(S)$ and $D(R)$, so that the sequence $S \rightarrow D_{\phi}(T) \rightarrow D(R) \rightarrow 0$ is exact. This sequence corresponds, under the functor $\operatorname{Hom}_{R}(-, M)$ to the sequence (2.1).

Finally, we will define a $K$-linear (Z-linear in Case G) map $\theta: D_{\phi}(T) \rightarrow S$ such that $\theta j$ is the identity on $S$. Hence the sequence

$$
0 \rightarrow S \rightarrow D_{\phi}(T) \rightarrow D(R) \rightarrow 0
$$

is 2 -exact. The above mentioned isomorphism of functors gives

$$
\operatorname{Ext}_{R}^{n}\left(D_{\phi}(T), M\right) \cong H^{n}(T, M),
$$

so that the desired result follows from the existence and properties of the connecting homomorphism for Ext.

Case A. $R \otimes_{K} T \otimes_{K} R$ is given the natural $R$-module structure, and $D_{\phi}(T)$ is the quotient by the submodule generated by

$$
\{\phi(x) \otimes y \otimes 1-1 \otimes x y \otimes 1+1 \otimes x \otimes \phi(y) \mid x, y \in T\} .
$$

The map from $S$ to $R \otimes T \otimes R$ which sends $s$ to $1 \otimes s \otimes 1$ induces $j$. Let $\alpha: R \rightarrow T$ 
be a $K$-splitting, so that $\phi \alpha=$ identity. Note that $\alpha(x) y-\alpha(x \phi(y)) \in S$ for $x \in R$ $y \in T$. Then $\theta$ is induced by the map from $R \otimes T \otimes R$ to $S$ which sends $x \otimes y \otimes z$ to $(\alpha(x) y-\alpha(x \phi(y)) z$.

Case S. $D_{\phi}(T)$ is the quotient of $R \otimes_{K} T$ by the submodule generated by $\{\phi(x) \otimes y-1 \otimes x y+\varepsilon(y) \otimes x \mid x, y \in T\}$ where $\varepsilon: T \rightarrow K$ is the augmentation. The map sending $s$ to $1 \otimes s$ induces $j$. The class of $x \otimes y \in R \otimes T$ is sent to $\alpha(x) y-\alpha(x \phi(y))$ by $\theta$.

Case G. Let $Z[R \times T]=Z[R] \otimes Z[T]$ be the group algebra on the product group $R \times T$, with the natural $R$-module structure. Let $D_{\phi}(T)$ be the quotient by the subgroup generated by $\{\phi(x) \otimes y-1 \otimes x y+1 \otimes x \mid x, y \in T\}$. The natural injection induces $j$ as usual, and $\theta$ is induced by the map sending $x \otimes y$ to $\alpha(x) y[\alpha(x \phi(y))]^{-1}$, where $\alpha: R \rightarrow T$ is any set-theoretic map such that $\phi \alpha=$ identity.

Case L. Assume first that $R$ is $K$-free and let $R^{e}$ be the Universal Enveloping Algebra of $R$. Then, by the Birkhoff-Witt Theorem [1, XIII, §3] $R$ is contained in $R^{e}$, and $R^{e}$ is $K$-free on a basis consisting of 1 and all elements of the form $x_{1} \cdots x_{n}$, where $n \geqq 1$ and $x_{1} \leqq \cdots \leqq x_{n}$ are elements of a linearly ordered $K$-basis for $R$. $D_{\phi}(T)$ is the quotient of $R^{e} \otimes_{K} T$ by the submodule generated by $\{\phi(x) \otimes y-1 \otimes x y-\phi(y) \otimes x \mid x, y \in T\}$. The map which sends $s$ to $1 \otimes s$ induces $j$. We have $R^{e} \otimes T=R^{e} \otimes S \oplus R^{e} \otimes \alpha(R)$. We will define a map $\mu: R^{e} \otimes T \rightarrow S$, and $\theta$ will be induced by $\mu$. The $R$-module structure of $S$ yields an $R^{e}$-module structure, and we use this to define $\mu$ on $R^{e} \otimes S$ such that $\mu(u \otimes s)=u s$ for $u \in R^{e}, s \in S . R^{e} \otimes \alpha(R)$ has a $K$-basis consisting of elements $1 \otimes \alpha(y)$ and $x_{1} \cdots x_{n} \otimes \alpha(y)$ with $y$ and $x_{1} \leqq \cdots \leqq x_{n}$ elements of the $K$-basis of $R$. Define $\mu(1 \otimes \alpha(y))=0$, and $\mu\left(x_{1} \cdots x_{n} \otimes \alpha(y)\right)=0$ whenever $x_{n} \leqq y$. Let $R_{n}^{e}$ be the $K$-submodule of $R^{e}$ generated by products of $\leqq n$ elements of $R$. We may complete the definition of $\mu$, using induction on $n$, by requiring

$$
\mu\left(x_{1} \cdots x_{n} \otimes \alpha(y)\right)=\mu\left(x_{1} \cdots x_{n-1} \otimes \alpha\left(x_{n}\right) \alpha(y)\right)+\mu\left(x_{1} \cdots x_{n-1} y \otimes \alpha\left(x_{n}\right)\right)
$$

whenever $y<x_{n}$; because, choosing $i$ such that $x_{i-1} \leqq y<x_{i}$, we have $x_{1} \cdots x_{n-1} y-x_{1} \cdots x_{i-1} y x_{i} \cdots x_{n-1} \in R_{n-1}^{e}$, so that both terms on the right may be taken as already defined.

It is now sufficient to show that $\mu$ vanishes on the requisite submodule, since it is then immediate that $\theta$ will have the desired property. We therefore must show

$$
\begin{gathered}
\mu(u \phi(z) \otimes s)=\mu(u \otimes z s), \quad u \in R^{e}, \quad z \in T, \quad s \in S, \\
\mu\left(x_{1} \cdots x_{n} y \otimes \alpha(z)\right)=\mu\left(x_{1} \cdots x_{n} \otimes \alpha(y) \alpha(z)\right)+\mu\left(x_{1} \cdots x_{n} z \otimes \alpha(y)\right), \\
y, z \text { and } x_{1} \leqq \cdots \leqq x_{n} \text { elements of the } K \text {-basis of } R,
\end{gathered}
$$

where in (2.3) we allow $n=0$, with the obvious meaning.

Equation (2.2) follows at once from the definition of $\mu$. If we assume inductively 
that equation (2.3) has been shown for all values of $n$ smaller than a given one then, combining with (2.2), we obtain

$$
\mu(u \phi(y) \otimes z)=\mu(u \otimes y z)+\mu(u \phi(z) \otimes y), \quad u \in R_{n-1}^{e}, \quad y, z \in T .
$$

In what follows, we will denote $x_{1} \cdots x_{n-1}$ by $x$, and multiplication in $R$ by the customary Lie brackets. We wish to establish (2.3). If $x_{n} \leqq y$ or $x_{n} \leqq z$, it holds by definition. If not, choose $i$ such that $x_{i-1} \leqq y<x_{i}$. We have

$$
x x_{n} y \otimes \alpha(z)=x y x_{n} \otimes \alpha(z)+x\left[x_{n}, y\right] \otimes \alpha(z)
$$

and since $x y-x_{1} \cdots x_{i-1} y x_{i} \cdots x_{n-1} \in R_{n-1}^{e}$ we may use (2.4) and the definition of $\mu$ to conclude from this that

$$
\begin{aligned}
\mu\left(x x_{n} y \otimes \alpha(z)\right)= & \mu\left(x y \otimes \alpha\left(x_{n}\right) \alpha(z)\right)+\mu\left(x y z \otimes \alpha\left(x_{n}\right)\right) \\
& +\mu\left(x \otimes\left(\alpha\left(x_{n}\right) \alpha(y)\right) \alpha(z)\right)+\mu\left(x z \otimes \alpha\left(x_{n}\right) \alpha(y)\right) .
\end{aligned}
$$

Combining (2.5) with the equation obtained from it by the symmetry of $y$ and $z$, and using the Jacobi identity, we conclude

$$
\begin{aligned}
\mu\left(x x_{n} y \otimes\right. & \alpha(z))-\mu\left(x x_{n} z \otimes \alpha(y)\right) \\
& =\mu\left(x[y, z] \otimes \alpha\left(x_{n}\right)\right)+\mu\left(x \otimes(\alpha(z) \alpha(y)) \alpha\left(x_{n}\right)\right) .
\end{aligned}
$$

But the right-hand side of this may be transformed, by applying (2.4) to its first term, into $\mu\left(x x_{n} \otimes \alpha(y) \alpha(z)\right)$, thus yielding (2.3), and completing the demonstration.

More generally, since $R$ is $K$-projective there is a $K$-module $R^{\prime}$ such that $R \oplus R^{\prime}$ is $K$-free. Consider $R^{\prime}$ as an abelian Lie algebra. Then the mapping $\phi \times 1: T \times R^{\prime} \rightarrow R \times R^{\prime}$ of product algebras is a surjection with kernel $S \times\{0\}=S$, and the $K$-module $R \times R^{\prime}=R \oplus R^{\prime}$ is free. Hence by the above the natural map from $S$ to $D_{\phi \times 1}\left(T \times R^{\prime}\right)$ is $K$-split. The Lie algebra homomorphism from $T$ to $T \times R^{\prime}$ which sends $y$ to $y \times 0$ induces an algebra homomorphism from $T^{e}$ to $\left(T \times R^{\prime}\right)^{e}$ and thus a $K$-linear map from $D_{\phi}(T)$ to $D_{\phi \times 1}\left(T \times R^{\prime}\right)$ such that the diagram

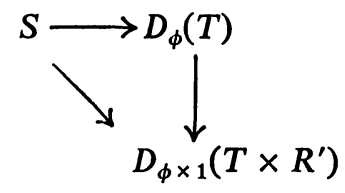

commutes. Thus we also obtain a $K$-splitting for the intap from $S$ to $D_{\phi}(T)$.

3. Extensions. If $R \in \mathscr{C}$, a singular extension of $R$ is a surjection $\phi: T \rightarrow R$ with kernel $S$ such that $S^{2}=0$, allowing us to consider $S \in \mathscr{M}_{R}$. In Cases A and $S$ we require $\phi$ to be $K$-split, and in Case $\mathrm{L}$ we require $R$ to be $K$-projective. If $R \in \mathscr{C}$, $S \in \mathscr{M}_{R}$, an extension of $R$ by $S$ is a singular extension $\phi: T \rightarrow R$ together with a map $\psi: S \rightarrow T$ which is an $R$-linear isomorphism of $S$ onto the kernel of $\phi$. 
As usual, two such extensions $(\psi, T, \phi)$ and $\left(\psi^{\prime}, T^{\prime}, \phi\right)$ are said to be equivalent in case there is a commutative diagram

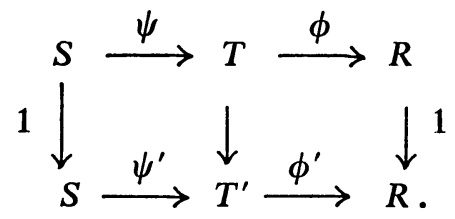

We introduce the notion of Baer multiplication of equivalence classes and show that they form a group, $\mathscr{M}(R, S)$, isomorphic in a natural way to $H^{1}(R, S)$. The ideas parallel those of $[1, \mathrm{XIV}, \S 1]$.

Given two extensions $(\psi, T, \phi)$ and $\left(\psi^{\prime}, T^{\prime}, \phi\right)$ we define their Baer composition $(\psi, T, \phi) *\left(\psi^{\prime}, T^{\prime}, \phi^{\prime}\right)$ as follows. $U=\left\{\left(t, t^{\prime}\right) \in T \times T^{\prime} \mid \phi(t)=\phi^{\prime}\left(t^{\prime}\right)\right\}$ and $I=\left\{\left(\psi(s), \psi^{\prime}(-s)\right) \mid s \in S\right\}$. Then it is easily seen that $U$ is a subobject of $T \times T^{\prime}$ and $I$ is an invariant subobject of $U$. We define $T * T^{\prime}=U / I$. Let $\pi: U \rightarrow T * T^{\prime}$ be the natural projection. Define $\psi * \psi^{\prime}: S \rightarrow T * T^{\prime}$ by $\psi * \psi^{\prime}(s)=\pi(\psi(s), 0)$ $=\pi\left(0, \psi^{\prime}(s)\right)$ and $\phi \times \phi^{\prime}: U \rightarrow R$ by $\phi \times \phi^{\prime}\left(t, t^{\prime}\right)=\phi(t)=\phi^{\prime}\left(t^{\prime}\right)$. Since $\phi \times \phi^{\prime}(I)=0$, there is a map $\phi * \phi^{\prime}: T * T^{\prime} \rightarrow R$ with $\phi * \phi^{\prime} \circ \pi=\phi \times \phi^{\prime}$. It is readily checked that $\left(\psi * \psi^{\prime}, T * T^{\prime}, \phi * \phi^{\prime}\right)$ is an extension of the desired type. We define $(\psi, T, \phi) *\left(\psi^{\prime}, T^{\prime}, \phi^{\prime}\right)=\left(\psi * \psi^{\prime}, T * T^{\prime}, \phi * \phi^{\prime}\right)$. Let $\left(\psi_{0}, T_{0}, \phi_{0}\right)$ be the extension in which $T_{0}=S \times R$ (Definition 1.1). It is a straightforward exercise to show

Proposition 3.1. The composition * is defined on equivalence classes and makes $\mathscr{M}(R, S)$ into an associative monoid whose identity element is the class containing $\left(\psi_{0}, T_{0}, \phi_{0}\right)$.

PROPOSITION 3.2. There is a natural monoid morphism

$$
\Delta: \mathscr{M}(R, S) \rightarrow H^{1}(R, S)
$$

Proof. Let $F=F(R)$ be the free object over $R$ as in Proposition 1.2 and consider the exact sequence $0 \rightarrow M \rightarrow{ }^{\beta} F \rightarrow{ }^{\alpha} R \rightarrow 0$ in which $\alpha: F \rightarrow R$ is the natural surjection, $M$ is its kernel and $\beta: M \rightarrow F$ is the inclusion. Given an extension $(\psi, T, \phi)$ we can use the adjointness relationship satisfied by $F$ to find a map $\tau: F \rightarrow T$ whose restriction to $M$ induces a map $v: M \rightarrow S$ so that the following diagram is commutative.

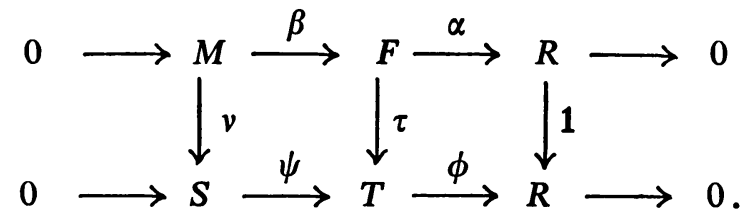


Moreover, $v\left(M^{2}\right)=0$. Hence $v$ induces a map $v^{\prime}: M / M^{2} \rightarrow S$ which is easily seen to be an $R$-homomorphism.

The canonical injection of $R$ into $F(R)$ yields a $K$-splitting for $\alpha$, so that we have a connecting homomorphism $\theta: \operatorname{Hom}_{R}\left(M / M^{2}, S\right) \rightarrow H^{1}(R, S)$. Similarly, we obtain $\Theta: \operatorname{Hom}_{R}(S, S) \rightarrow H^{1}(R, S)$. Moreover, since $H_{\alpha}^{1}(F, S)=0$ (Proposition 1.2), $\theta$ is a surjection. Hence we obtain a commutative and exact diagram

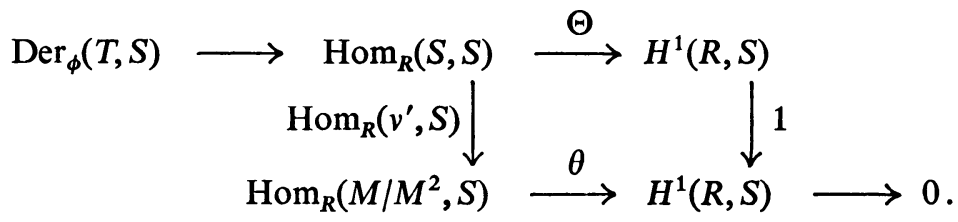

We let $\Delta(\psi, T, \phi)=\Theta\left(v^{\prime}\right)=\theta\left(1_{s}\right)$. The latter equality shows that this does not depend on the choice of $\tau$. Moreover it is clear that $\Delta$ is really defined on equivalence classes. That $\Delta$ is a monoid morphism now follows from

Lemma 3.5. Let $(\psi, T, \phi),\left(\psi^{\prime}, T^{\prime}, \phi^{\prime}\right)$ be two extensions and let $\tau, v, \tau^{\prime}, v^{\prime}$ be chosen as in (3.3). Then there is a map $\tau * \tau^{\prime}: F \rightarrow T * T^{\prime}$ so that the following diagram is commutative.

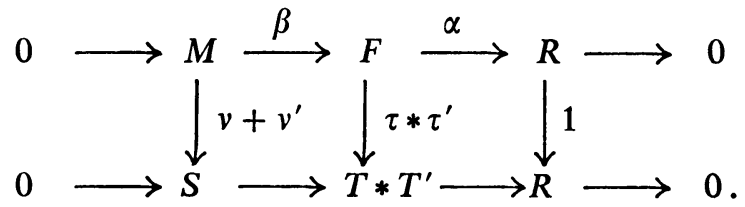

Proof. Let $\pi: U \rightarrow T * T^{\prime}$ be as before and define $\tau * \tau^{\prime}(x)=\pi\left(\tau(x), \tau^{\prime}(x)\right)$, $x \in F$.

Proposition 3.6. $\Delta$ is an epimorphism.

In fact, since $\theta$ is an epimorphism, it suffices to show that if $v: M \rightarrow S$ is such that $v\left(M^{2}\right)=0$ and the induced map $v^{\prime}: M / M^{2} \rightarrow S$ is $R$-linear, then we can find an extension $(\psi, T, \phi)$ making (3.3) commutative. Let $V=S \times F$ as a set in Case $\mathrm{G}$ and a $K$-module in the other cases, with multiplication defined by $(s, x)\left(s^{\prime}, x^{\prime}\right)$ $=\left(\alpha(x) s^{\prime}+s \alpha\left(x^{\prime}\right), x x^{\prime}\right) x, x^{\prime} \in F, s, s^{\prime} \in S$. Let $J=\{(v(m), \beta(-m)) \mid m \in M\}$. Then $J$ is an invariant subobject. Let $T=V / J$ and $\rho: V \rightarrow T$ be the natural projection. Define $\psi: S \rightarrow T$ by $\psi(s)=\rho(s, 0)$ and $\phi^{\prime}: V \rightarrow R$ by $\phi^{\prime}(s, x)=\alpha(x)$, $x \in F, s \in S$. Then $\phi^{\prime}(J)=0$ and so $\phi^{\prime}$ induces a map $\phi: T \rightarrow R$ with $\phi \rho=\phi^{\prime}$. Map $\tau: F \rightarrow T$ by $\tau(x)=\rho(0, x)$. Then $(\psi, T, \phi)$ is the desired extension.

Proposition 3.7. $\Delta^{-1}(0)$ contains only the class of $\left(\psi_{0}, T_{0}, \phi_{0}\right)$.

Proof. Suppose $\Delta(\psi, T, \phi)=0$. Referring to (3.4), we conclude that there exists $d \in \operatorname{Der}_{\phi}(T, S)$ such that $d \psi$ is the identity. As remarked in Definition 1.1, 
$d$ corresponds to a morphism $f: T \rightarrow T_{0}$; and $f$ has the property that $f \psi=\psi_{0}$ and $\phi_{0} f=\phi$. Hence $T$ and $T_{0}$ are equivalent.

LEMMA 3.8. Let $N$ be a monoid, $G$ a group and $f: N \rightarrow G$ a monoid surjection with $f^{-1}(0)=0$. Then $f$ is an isomorphism (and $N$ is a group).

Proof. Trivial.

This completes the proof of

THEOREM 3.9. $\Delta: \mathscr{M}(R, S) \rightarrow H^{1}(R, S)$ is an isomorphism.

More generally, we may consider sequences

$$
E \quad 0 \longrightarrow S \stackrel{\psi}{\longrightarrow} E_{1} \longrightarrow \cdots \longrightarrow E_{n-1} \stackrel{\gamma}{\longrightarrow} T \stackrel{\phi}{\longrightarrow} R \longrightarrow 0 \quad(n>1)
$$

in which $\phi$ is a morphism in $\mathscr{C}(K$-split in all but Case $\mathrm{G})$ with kernel $V$, such that $V^{2}=0$; and such that the image of $\gamma$ is $V$, and, if $V$ is given the induced $R$ module structure,

$$
0 \longrightarrow S \longrightarrow E_{1} \longrightarrow \cdots \longrightarrow E_{n-1} \longrightarrow V \longrightarrow 0
$$

is an exact sequence of $R$-modules ( $K$-split in all but Case G). In Case $L$ we must also assume that $R$ is $K$-projective.

Two such sequences $E$ and $E^{\prime}$ are related if there is a commutative diagram

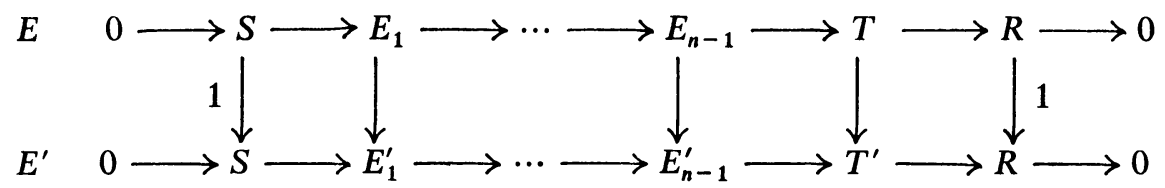

and we consider $n$-fold extensions of $R$ by $S$ to be equivalence classes of such sequences under the equivalence relation generated by this relation.

Given sequences $E$ and $E^{\prime}$, their Baer composition is the sequence

$$
E * E^{\prime} \quad 0 \rightarrow S \rightarrow E_{1} * E_{1}^{\prime} \rightarrow E_{2} \oplus E_{2}^{\prime} \rightarrow \cdots \rightarrow E_{n-1} \oplus E_{n-1}^{\prime} \rightarrow T * T^{\prime} \rightarrow R \rightarrow 0,
$$

where $E_{1} * E_{1}^{\prime}=E_{1} \oplus E_{1}^{\prime} /\left\{\left(\psi(s), \psi^{\prime}(-s)\right) \mid s \in S\right\}$ and $T * T^{\prime}=\left\{\left(t, t^{\prime}\right) \in T \times T^{\prime} \mid \phi(t)\right.$ $\left.=\phi^{\prime}\left(t^{\prime}\right)\right\}$ and the morphisms are the obvious ones. This clearly defines a product on equivalence classes.

Given a sequence $E$ we obtain an iterated connecting homomorphism

$$
\operatorname{Hom}_{R}(S, S) \rightarrow \operatorname{Ext}_{R}^{n-1}(V, S) \rightarrow H^{n}(R, S)
$$

and we associate with $E$ the image in $H^{n}(R, S)$ under this iteration of the identity morphism $1_{s}$. This defines a map $\Delta_{n}$ from $n$-fold extensions to $H^{n}(R, S)$.

THEOREM 3.10. $\Delta_{n}$ is an isomorphism of groups.

Proof. We define its inverse $\Phi$. Let $F=F(R)$, and let $M=\bar{M} / \bar{M}^{2}, F=F / \bar{M}^{2}$, 
where $\bar{M}$ is the kernel of the canonical surjection $\alpha: F \rightarrow R$. We have an exact sequence

$$
0=H^{n-1}(F, S) \rightarrow \operatorname{Ext}_{R}^{n-1}(M, S) \rightarrow H^{n}(R, S) \rightarrow H^{n}(F, S)=0
$$

by Proposition 1.2 and Theorem 2.2. Given an element $a \in H^{n}(R, S)$, we obtain $b \in \operatorname{Ext}_{R}^{n-1}(M, S)$ by the isomorphism (3.11). This in turn [4] yields an extension

$$
0 \longrightarrow S \longrightarrow E_{1} \longrightarrow \cdots \longrightarrow E_{n-1} \longrightarrow M \longrightarrow 0
$$

which is uniquely determined up to equivalence by the property that $b$ is the image of the identity under the corresponding iterated connecting homomorphism from $\operatorname{Hom}_{R}(S, S)$ to $\operatorname{Ext}_{R}^{n-1}(M, S)$. Let $\Phi(a)$ be the class of extension

$$
0 \longrightarrow S \longrightarrow E_{1} \longrightarrow \cdots \longrightarrow E_{n-1} \longrightarrow F \longrightarrow R
$$

obtained by combining (3.12) with $0 \rightarrow M \rightarrow F \rightarrow R \rightarrow 0$. Clearly $\Delta_{n} \Phi$ is the identity. One can also show that $\Phi \Delta_{n}$ is the identity, and that $\Phi$ is additive, which completes the proof.

Theorem 3.10 suggests that $H(R, N)$ can be obtained as the homology of the complex

$\cdots \longleftarrow \operatorname{Hom}_{R}\left(X_{n}, N\right) \longleftarrow \cdots \longleftarrow \operatorname{Hom}_{R}\left(X_{1}, N\right) \longleftarrow \operatorname{Der}(F, N) \longleftarrow 0$

where $0 \rightarrow M \rightarrow F \rightarrow R \rightarrow 0$ is as in the proof of the theorem, and $\cdots \rightarrow X_{n} \rightarrow$ $\cdots \rightarrow X_{1} \rightarrow M \rightarrow 0$ is a renumbered projective resolution of $M$ (in the sense dual to the notion of $\mathscr{Q}$-injective resolution). Indeed, the connecting homomorphism for the second variable is easily seen to exist and produce an exact sequence as usual. Moreover, these functors vanish in positive degree for $N \mathscr{Q}$-injective, as is seen by using (2.1.1). Since these properties characterize the functors $H^{n}(R,-)$, the result follows.

The idea for this definition of cohomology by means of a resolution of $R$ is essentially contained in [6] and will be exploited in [5].

4. Free products of groups. If $G_{1}$ and $G_{2}$ are groups, let $G_{1} \circ G_{2}$ denote their free product. If $H$ is any group, $G_{1} \circ G_{2}$ is characterized by a natural isomorphism

$$
\operatorname{Hom}\left(G_{1} \circ G_{2}, H\right) \cong \operatorname{Hom}\left(G_{1}, H\right) \times \operatorname{Hom}\left(G_{2}, H\right)
$$

where "Hom" denotes group homomorphisms.

THEOREM 4.1. If $M$ is simultaneously a $G_{1}$-and a $G_{2}$-module, and thus a $G_{1} \circ G_{2}$-module, then the natural mapping from $H^{n}\left(G_{1} \circ G_{2}, M\right)$ to $H^{n}\left(G_{1}, M\right)$ $\oplus H^{n}\left(G_{2}, M\right)$ is an isomorphism for every $n$.

Proof. If $G$ is a group and $Q$ is an injective $Z$-module, then $\operatorname{Hom}_{Z}(Z[G], Q)$ $\cong \operatorname{Map}(G, Q)$ is an injective $G$-module, where "Map" denotes set mappings, 
and, for $f \in \operatorname{Map}(G, Q), x, y \in G,(x \cdot f)(y)=f(y x)$. If $G^{\prime} \subset G$ and $G=\bigcup_{i} x_{i} G^{\prime}$ is a coset decomposition, then we have a $G^{\prime}$-module isomorphism $\operatorname{Map}(G, Q)$ $\cong \prod_{i} \operatorname{Map}\left(x_{i} G^{\prime}, Q\right)$, so that $\operatorname{Map}(G, Q)$ is simultaneously a $G^{\prime}$-injective and a $G$-injective module. Given a $G$-module $M$ we choose a $Z$-injective $Q$ with $M \subset Q$, and define a $G$-linear injection from $M$ into $\operatorname{Map}(G, Q)$ by sending $m$ to $f_{m}$, where $f_{m}(x)=x m$. Thus every $G$-module can be imbedded in a module which is injective as a module over every subgroup of $G$.

Applying this to the situation under consideration, we see that there is a simultaneous $G_{1^{-}}, G_{2^{-}}$, and $G_{1} \circ G_{2^{-}}$-injective resolution, $Y$, of $M$. From (1) and the correspondence given in Definition 1.1 we obtain

$$
\operatorname{Der}\left(G_{1} \circ G_{2}, Y\right) \cong \operatorname{Der}\left(G_{1}, Y\right) \oplus \operatorname{Der}\left(G_{2}, Y\right),
$$

and passing to homology yields the desired result.

\section{REFERENCES}

1. H. Cartan and S. Eilenberg, Homological algebra, Princeton Univ. Press, Princeton, N. J., 1956.

2. S. Eilenberg and J. Moore, Foundations of relative homological algebra, Mem. Amer. Math. Soc. No. 55 (1965), 39 pp.

3. G. Hochschild, Relative homological algebra, Trans. Amer. Math. Soc. 82 (1956), 246-269.

4. N. Yoneda, On the homology theory of modules, J. Fac. Sci. Univ. Tokyo Sect. 18 (1954), 193-227.

5. M. Barr, Cohomology theory for commutative algebras. I, II, Proc. Amer. Math. Soc. 16 (1965), 1378-1390.

6. M. Gerstenhaber, A uniform cohomology theory for algebras, Proc. Nat. Acad. Sci. U.S.A. 51 (1964), 626-629.

COLUMBia UNIVERSITY,

New York, New YoRK 